\title{
Círculos literarios: el poder de la lectura en el desarrollo de competencias
}

CARMEN PÉREZ MARTÍNEZ

Universitat Politècnica de València

mapem13m@upvnet.upv.es

Resumen: El objetivo de este artículo es demostrar la utilidad de los círculos literarios a la hora de desarrollar la competencia comunicativa y las interrelacionadas a ella, como son la lingüística, sociolingüística, discursiva y estratégica, en una actividad que permite al alumno no solo desinhibirse de la formalidad del ámbito académico, sino disfrutar de la literatura en la lengua de aprendizaje. También pone en práctica una conciencia crítica que servirá para trabajar estructuras gramaticales relacionadas con la opinión.

Palabras clave: círculo de lectura, club de lectura, tertulia dialógica, competencias.

\section{Literature Circles: the power of Reading in developing skills}

Abstract: The main purpose of this article is to demonstrate the usefulness of literature circles when developing communicative competence and those related to it, such as linguistic, sociolinguistic, discursive and strategic competence, in an activity that allows the learner not only to uninhibited from the formality of the academic environment but also to enjoy literature in the language of learning. It also puts into practice a critical awareness that will serve to work on grammatical structures related to opinion.

Key words: literature circles, reading club, dialogic literary circle, competences.

\section{Introducción}

Es indiscutible que los cuentos nos abren la puerta a la literatura. Con ellos aprendemos valores, emociones, modelos de comportamiento e, incluso, como sugiere Álvarez (2011), ritos iniciáticos. Poco a poco, esos relatos que alientan nuestro crecimiento personal y que suponen un reflejo de aspectos de nosotros mismos y de nuestra complejidad humana (Martínez, 2017: 17-18) evolucionan hacia estructuras más complejas: las obras literarias. Sin embargo, a pesar de todo ese bagaje emocional y cultural, llegamos a la adolescencia y esas obras se convierten en un mero indicador para comprobar el alcance de nuestra comprensión lectora. Por tanto, la cuestión aquí es: ¿por qué ese encorsetamiento didáctico cuando trabajamos la lectura en el aula? Y en definitiva, lo que debemos plantearnos es: ¿qué podemos hacer para transformar las lecturas de simple instrumento a un recurso mediante el cual canalizar las diferentes competencias de nuestros alumnos? En nuestra opinión, la respuesta es evidente: trabajar con círculos de lectura.

2. Los círculos literarios y el Marco común europeo de referencia para las lenguas 
Por supuesto, cualquier actuación dentro del aula debe acogerse a un marco teórico que dé sentido y coherencia al contenido que pretendemos enseñar. En el caso de segundas lenguas, nos regimos a partir del Marco común europeo de referencia para las lenguas. Y lo que este nos dice es que, para «realizar las tareas y las actividades que se requieren para abordar las situaciones comunicativas en las que se ven envueltos, los usuarios y los alumnos utilizan varias competencias desarrolladas en el curso de su experiencia previa» (Instituto Cervantes, 2002). Evidentemente, la participación del alumno en situaciones comunicativas hace que, de manera directa e indirecta, desarrolle todas las competencias, tanto generales como comunicativas. En este sentido, el círculo literario, como actividad comunicativa, también cumple con esta premisa, si bien es verdad que en algunos casos de una manera más directa o indirecta.

Dentro de las competencias generales, está claro que el alumno debe desarrollar un conocimiento declarativo (saber) que le viene marcado por la lectura de un texto hispánico, inscrito dentro de la sociedad y cultura de la comunidad en la que se habla el idioma que está aprendiendo. Pero también actúa de acuerdo con unas destrezas y habilidades (saber hacer) que le permiten relacionar su cultura de origen con la cultura extranjera, superando de este modo imágenes estereotipadas. La competencia "existencial" (saber ser) viene filtrada por la propia subjetividad del alumno y su particular forma de abordar el texto, a partir de su actitud, motivación, valores, creencias, estilos cognitivos y personalidad. Por último, la capacidad de aprender (saber aprender) está determinada tanto por el trabajo individual que cada estudiante debe hacer a la hora de preparar su papel en cada sesión de una manera independiente y autodirigida, como por la reconstrucción de significado que realiza en colaboración con los otros participantes que le ayudan a paliar lagunas interpretativas y a amplificar su lectura con otras perspectivas.

En cuanto a las competencias comunicativas, el alumno debe hacer uso de los recursos lingüísticos (tanto oralmente como por escrito) y desarrollar tanto la dimensión social, al tratarse de una actividad académica que requiere el manejo de una serie de reglas de cortesía y registros, como la pragmática, determinada por su condición discursiva y su naturaleza interactiva y transaccional (Instituto Cervantes, 2002: capítulo 5). Asimismo, no podemos olvidar la competencia literaria como elemento intrínseco de esa competencia comunicativa, dado que prescindir de ella en el proceso de enseñanzaaprendizaje «impide o desacelera el desarrollo de ciertas habilidades lecto-literarias y, por ende, el desarrollo completo de la competencia comunicativa» (García, 2017: 2).

\section{Los círculos literarios en el aula}

\subsection{Círculos literarios: qué es y qué no es}

Existen muchas denominaciones para referirse a la actividad en la que un grupo de personas se reúne para hablar sobre un libro: círculo literario, club de lectura, grupo de lectura... incluso podríamos seguir añadiendo nombres solo jugando con las palabras: club literario, círculo de lectura... En cualquier caso, la pregunta sería: ¿son iguales todas estas propuestas? Y en caso de que no sea así, ¿cuál es la diferencia?

Para arrojar un poco de luz sobre el asunto, Daniels (2002: 1) y veinte profesores más pertenecientes a diferentes niveles educativos decidieron reunirse en 1993 para escribir un libro sobre lo que ellos llamaron "círculos literarios". Durante su investigación, se 
dieron cuenta de que dotando a los alumnos de libertad de elección, tiempo, responsabilidad, una mínima dirección y una estructura de trabajo, estos eran capaces de actuar como buenos lectores, es decir, de leer buenos libros, pensar en ellos con la suficiente profundidad y formar parte de discusiones literarias de forma animada y documentada. Básicamente, Daniels y sus colegas definían de esta manera los círculos literarios:

Literature circles are small, peer-led discussion groups whose members have chosen to read the same story, poem, article, or book. While reading each group-assigned portion of the text (either in or outside of class), members make notes to help them contribute to the upcoming discussion, and everyone comes to the group with ideas to share. Each group follows a reading and meeting schedule, holding periodic discussions on the way through the book. When they finish a book, the circle members may share highlights of their reading with the wider community; then they trade members with other finishing groups, select more reading, and move into a new cycle. (Daniels, 2002: 2)

Sin embargo, también reconocían que en todas partes del mundo hay profesores que están aplicando versiones personalizadas de lo que ellos llaman "clubs de lectura".

Por tanto, la diferencia principal entre un club de lectura y un círculo literario sería que el primero es una reunión entre adultos en la que cada uno opina sobre el libro, mientras que el segundo se lleva a cabo entre niños y jóvenes con una estructura y una organización determinadas.

\subsection{Los círculos literarios como práctica educativa}

No hace falta remarcar la importancia y el valor que las diferentes lecturas tienen en nuestra vida, sobre todo como adultos. El problema viene cuando intentamos introducirlas en un ámbito académico, donde no gozan de la misma acogida. Para realizar cualquier actividad en el aula, la motivación es fundamental, y más si cabe cuando hablamos de lecturas obligatorias. Como afirman Muñoz, Valenzuela, Avendaño y Núñez (2016: 53) en su artículo: «un estudiante motivado por la lectura de textos pone al servicio del aprendizaje todas sus herramientas cognitivas, sociales y afectivas en la consecución de su objetivo de aprendizaje». Por lo tanto, la elección de una actividad que les permita construir su propio aprendizaje, incidiendo en aquellas estrategias implicadas de forma directa (metacognitivas o cognitivas) o indirecta (sociales y comunicativas) en dicho proceso (Miñano, 2000), junto con una adecuada animación lectora, convierten los círculos literarios en una opción idónea para trabajar las lecturas de clase.

A propósito de la propuesta de Daniels (2002), compartimos la adaptación que Furr (2004) hace de sus círculos literarios, empezando por replantear algunas de sus características principales. De esta manera, si para Daniels era necesario que los estudiantes eligiesen las obras, se organizasen en pequeños grupos de acuerdo con el libro elegido (diferente en cada caso) y que, una vez acabada la lectura, la compartiesen y acometiesen la formación de nuevos grupos para una nueva lectura, Furr (2004: 4-5) sugiere estas modificaciones para la clase de idiomas:

1. Instructors select materials appropriate for their student population.

2. Small temporary groups are formed, based on student choice or the Instructor's discretion.

3. Different groups are usually reading the same text.

4. When books are finished, readers may prepare a group project and/or the Instructor may provide additional information to "fill in some of the gaps" in student understanding--I call this step backloading the instruction. 
After the group projects or additional instruction, new groups are formed, based on student choice or the Instructor's discretion.

Como podemos observar, la diferencia principal radica en el papel del docente a la hora de organizar la actividad de acuerdo con unos requisitos vinculados con el aprendizaje de una lengua. Así, seleccionar el material según el nivel de los alumnos, agrupar a los estudiantes buscando cierto equilibrio, trabajar el mismo texto o realizar proyectos posteriores a la lectura son prácticas habituales en el aula de lenguas que, en este caso, se ponen al servicio de los círculos literarios.

\section{Objetivos}

Junto a los objetivos estrictamente académicos que vienen exigidos por el Plan Curricular del Instituto Cervantes y que se pueden insertar en el desarrollo de la actividad (como por ejemplo, las estructuras de opinión y valoración), es esencial plantearse qué es lo que pretendemos conseguir con el círculo literario. En general, el objetivo que priorizamos sobre los demás cuando planteamos esta actividad es el de disfrutar de las obras literarias, sobre todo como instrumentos que vehiculan unas ideas, que pueden estar a favor o en contra de ese libro, pero que, en definitiva, lo que hacen es acercarlo al estudiante y predisponerlo para una lectura crítica. Además, no debemos olvidar que dichas obras son una vía perfecta para que el estudiante se acerque a la cultura española o hispanoamericana en alguno de sus aspectos (y, en este sentido, cobraría gran importancia la selección de las obras con unos fines concretos, como es el caso de los petroglifos y cultos ancestrales gallegos en Cartas de invierno, de Agustín Fernández Paz o, desde un punto de vista textual, el uso de la carta informal en esta misma obra). A todos estos objetivos, añadimos los relacionados con la mejora de la comprensión lectora y la expresión oral como destrezas principales, aunque está claro que también es necesario desarrollar la comprensión auditiva y, en determinados casos, la expresión escrita.

Un último objetivo que no obviamos por la trascendencia que tiene es la aceleración de los aprendizajes a través del aprendizaje basado en interacciones, dado que, como sostienen muchos autores, más que el entorno son estas últimas las que promueven el aprendizaje del individuo. "A través del diálogo las personas intercambian ideas, aprenden conjuntamente y producen conocimiento, encontrando y creando nuevos significados que transforman el lenguaje y el contenido de sus vidas» (Valls, Soler y Flecha, 2008: 73).

\section{Funcionamiento}

Para llevar a cabo esta actividad, hay que planificar el trabajo que se realizará antes y después de la sesión del círculo literario en sí, dado que pasa por diferentes fases. De esta manera, lo primero que hay que plantearse es el tiempo que va a necesitar el estudiante para preparar dicha sesión, que dependerá de aspectos como la extensión de la obra, la duración del curso o incluso la carga lectiva/profesional de los estudiantes, además de reflexionar sobre la misma o incluso evaluar su actuación y la de sus compañeros. A partir de ahí, se determinará la fecha de la reunión. En nuestro caso, suele ser mensual y las lecturas vienen determinadas por el nivel de los alumnos. Evidentemente, en un nivel A1A2 es muy complicado llevar a cabo una actividad que requiere una mínima destreza oral 
y lingüística; por lo tanto, lo ideal es efectuar estos círculos literarios a partir de un B1 en adelante.

En cuanto a la selección de las obras, como hemos comentado anteriormente, depende de diferentes factores, como la temática, su calidad literaria, el perfil y nivel de los alumnos, los aspectos lingüísticos o las cuestiones culturales que queramos desarrollar, entre otros. De este modo, si por ejemplo estamos trabajando el presente de indicativo y sus irregularidades, podríamos utilizar Sin noticias de Gurb, de Eduardo Mendoza, o La jaula de los gorilas, de Rodrigo Muñoz Avia. Dado que nuestro principal objetivo es fomentar el gusto del alumno por la obra literaria, nuestra elección se orienta más a textos de este tipo, si bien es cierto que se puede abordar cualquier tipología textual (expositiva, argumentativa, etc.). Además, teniendo en cuenta la heterogeneidad del alumnado, dicha elección debería ser variada, para abarcar a un mayor número de lectores. Actualmente, en literatura juvenil existe un amplio abanico de títulos que resultan idóneos en cuanto a vocabulario y contenido (tanto en narrativa como en teatro o poesía). Lo fundamental es elegir una obra completa con una historia que pueda alentar la discusión posterior (por ejemplo, El niño que vivía en las estrellas, de Jordi Sierra i Fabra, da pie a hablar sobre el maltrato infantil y sus diferentes tipos). Para clásicos de la literatura, sí que optamos por obras adaptadas, ya que el nivel de complejidad lingüística de algunas épocas dificultaría la comprensión para un alumno extranjero o poco avezado en ese estrato de la lengua, o con retoques ortotipográficos que las adecúen a la normativa actual de la Academia Española ${ }^{1}$.

Una última cuestión que se puede planificar y llevar a cabo previamente a la reunión es la relacionada con la animación lectora (sobre todo antes de que empiecen a leerse la obra), diseñando una serie de actividades que tengan como objetivo atraer la atención del estudiante hacia la obra o conocer algunos aspectos de su autor y su contexto. Estas actividades se basarían, por ejemplo, en inferir información a partir de los paratextos o la portada del libro; o el análisis de algunos fragmentos antes de su lectura, con el que Hernández (2019: 41-44) consigue profundizar en una obra de Almudena Grandes. También son interesantes las propuestas de Hernández (2018: 12-19) para investigar datos sobre el autor y su contexto, con actividades idóneas para trabajar determinados aspectos lingüísticos (por ejemplo, con la inclusión de huecos en una biografía extraída de Wikipedia) o microdestrezas de comprensión lectora (como la extracción de las ideas principales).

\subsection{Previamente a la sesión}

Los círculos literarios funcionan con roles, esto es, funciones que se asignan a cada estudiante para que desarrolle una forma de acercarse al libro. Su ventaja no solo radica en descentralizar la actividad y dotar al alumno tanto de mayor responsabilidad como de autonomía, sino que además facilita y estimula su participación en estos grupos (Daniels, 2002: 71). Además, el control en la actividad, al elegir libremente el material y la información (de acuerdo a unas pautas), junto con la generación de preguntas que van a formular a sus compañeros, fortalecen el compromiso con la lectura, «fomentando

\footnotetext{
1 Para clásicos de la literatura, recomendamos el catálogo de Vicens Vives (Aula de Literatura o Clásicos Hispánicos), cuyos trabajos introductorios y anotaciones a pie de página facilitan la comprensión de determinados aspectos de la vida cotidiana ahora inexistentes (por ejemplo: la figura del "Cobrador" en Historia de una escalera (1947-1948, de Antonio Buero Vallejo).
} 
asimismo la lectura sostenida y creando alumnos autónomos» (Buttiler y Massano, 2015: 6-7). Así, los estudiantes ganan confianza básicamente porque saben de lo que van a hablar (Furr, 2004).

En general, existen distintos tipos de roles que varían dependiendo del autor que los presente, si bien es verdad que podríamos perfilar unos roles básicos que, de alguna manera, son necesarios para desentrañar el significado de los libros. Y esto se debe, en gran medida, a que algunos de ellos tienen que ver con las estrategias de comprensión lectora. Eso sí, para que haya una adecuada transferencia de habilidades de una L1 a una L2 es necesario que compartan, al menos, el alfabeto romano (Furr, 2004). Y tampoco debemos subestimar las dificultades a las que se enfrentan los estudiantes ante textos literarios, donde, además de los problemas con el lenguaje poético, se experimentan otros relacionados con sus hábitos de atención, la lectura y su entrenamiento tanto en comprensión lectora como en pensamiento crítico (Sánchez, 2004: 75).

En cualquier caso, como bien señala Solé, «leer es un proceso de interacción entre el lector y el texto, proceso mediante el cual el primero intenta satisfacer [obtener una información pertinente para] los objetivos que guían su lectura» (1992: 21-22). Y esa finalidad hace que, frente a la misma información, se obtengan lecturas distintas. Por tanto, ahí es donde los roles juegan un papel fundamental, proporcionando perspectivismo y contribuyendo en la construcción colectiva de significado.

En este estudio en concreto, nos vamos a centrar en dos propuestas: la de Harvey (2002) y la de Oxford University Press (s.f.), dependiendo de si queremos abordar el libro desde una perspectiva literaria o más comunicativa.

Pues bien, para comenzar la actividad, es necesario crear los grupos de trabajo y repartir las fichas con los roles entre sus integrantes. El número de estos dependerá de la cantidad de estudiantes y los roles que vayamos a utilizar: si nos acogemos a la propuesta de Daniels (2002) o la de Oxford University Press (s.f.). En nuestro caso, el mínimo de alumnos con el que hemos puesto en marcha un círculo literario ha sido de cuatro estudiantes. No obstante, con un número menor se podría realizar perfectamente, adaptándolo a las exigencias de esa situación (por ejemplo, se podrían asignar dos o más roles a cada estudiante). Una vez agrupados, lo ideal es explicarles en qué consisten los roles y, a continuación, dar unos minutos para que cada uno de ellos, con su grupo, decida en común acuerdo cuál quiere desarrollar. Evidentemente, con esta actuación logramos, en primer lugar, un mayor compromiso por su parte (al tener poder de elección) y, en segundo lugar, una democratización del equipo de trabajo. Además, y esto es fundamental, vamos relegando el control en ellos y materializando el trabajo colaborativo y el modelo de aprendizaje centrado en el estudiante. Por último, quedaría consensuar el día en que se va a celebrar el círculo literario.

En cuanto a los roles ${ }^{2}$, en este estudio vamos a presentar dos:

1. A pesar de la gran variedad que propone, incluso dependiendo de su nivel educativo, Daniels (2002: 99-132) sugiere cuatro roles básicos que reflejan ciertas estrategias que los lectores usan habitualmente de manera consciente o inconsciente:

\footnotetext{
${ }^{2}$ La adaptación/traducción de los nombres es nuestra.
} 
a. Conector (Connector): relaciona lo que lee con su vida, sus sentimientos, sus experiencias, otros libros, autores, noticas, etc.

b. Analizador (Questioner): el encargado de analizar el texto, los personajes, la forma en que el autor muestra unos sentimientos, etc., o simplemente de motivar a sus compañeros o contraargumentar sus opiniones.

c. Maestro de los pasajes (Literary luminary/passage master): comparte pasajes que son especialmente importantes por algún motivo.

d. Ilustrador (Illustrator): nos recuerda la importancia de la visualización en la lectura, rememorando el libro a partir de gráficos, dibujos, fotografías o cualquier material no lingüístico.

Con ellos, se asegura cuatro formas diferentes de acercarse al texto: la analítica (questioner), la oral/dramática (literary luminary), la asociativa (connector) y la gráfica/artística (illustrator). Además, formula otros cuatro roles opcionales:

a. Resumidor (Summarizer): resume los puntos más importantes del relato.

b. Investigador (Researcher): presenta información relacionada con el contexto o con el tema del libro.

c. Mago de las palabras (Vocabulary enricher/word wizard): busca palabras interesantes de la lectura.

d. Rastreador de escenas (Travel tracer/scene setter): describe los lugares y ambientes por los que transcurren los personajes con palabras o en forma de mapa.

2. Por su parte, en su colección de libros Bookworms Club (Furr s.f.), desde Oxford University Press (s.f.) plantean seis, que en realidad serían el resultado de una adaptación de la propuesta de Daniels (dirigida a hablantes nativos que cursan la escuela elemental o secundaria) para las clases de EFL/ESL:

a. Guía de debate (Discussion leader): conduce el círculo literario, manteniendo a sus compañeros implicados con preguntas sobre el texto y asegurándose de que todos tienen la oportunidad de participar.

b. Resumidor (Summarizer): se encarga de encontrar los puntos clave de la historia (personajes, acontecimientos e ideas).

c. Conector (Connector): conecta la historia con el mundo y con sus propias experiencias.

d. Maestro de las palabras (Word master): elije aquellas palabras que son significativas para la historia.

e. Responsable de los pasajes (Passage person): localiza pasajes importantes, interesantes o complejos.

f. Coleccionista de cultura (Culture collector): señala las diferencias y similitudes de la cultura del libro con otras culturas.

Además, esta colección viene acompañada de una guía con instrucciones y claves para realizar un círculo de lectura con éxito (Furr 2011).

Como vemos, a pesar de sus puntos en común, el planteamiento de Daniels funciona mejor en una clase de literatura y el de Oxford University Press en una de enseñanza de lenguas, básicamente porque en el primer caso se focaliza en aspectos estructurales y temáticos del libro y, en el segundo, se incluyen aspectos como la interculturalidad con la figura del coleccionista de cultura (culture collector), lo cual permite al estudiante 
extranjero profundizar en el conocimiento de sociedades y culturas distintas a las suyas, acometiendo las competencias generales. Al mismo tiempo, este rol facilita a determinados alumnos establecer conexiones culturales y lingüísticas entre la lengua que se habla en su casa y la que aprenden en la escuela, como es el caso de los estudiantes latinos de primera generación nacidos en Estados Unidos, cuyo sistema educativo monolingüe en inglés prescinde de las experiencias culturales propias de su lengua materna (Rodríguez-Valls, 2008).

Aunque a la hora de preparar cada alumno su ficha debe formular diferentes preguntas dirigidas a promover la interacción entre sus compañeros y, lo más importante, a construir conjuntamente el significado del libro, lo cierto es que en algunos roles resulta más complicado desarrollar esta parte. En ese sentido, la propuesta de Boardman Moen (2004: 16) es muy interesante, dado que sugiere que cada estudiante escriba una pregunta para cada una de las siguientes categorías ${ }^{3}$ : Compara (compare), Contrasta (contrast), Describe (describe), Di por qué (why), Da ejemplos (give examples), Cómo lo sabes (how do you know), Qué significa (what does it means) y Opina-explica (opinion-explain), ampliando de esta manera el espectro interpretativo que se consigue al centrarse en un solo rol.

\subsection{Durante la sesión}

El desarrollo de la actividad debe durar como mínimo una hora para permitir que todos los integrantes puedan presentar sus roles a sus compañeros y, al mismo tiempo, puedan participar compartiendo sus opiniones sobre otros roles. En cuanto al máximo, depende del grupo y de la profundidad con que se hayan preparado los roles. Lo conveniente es hacer un seguimiento del trabajo de los estudiantes antes de la reunión para orientarles y corregir gramaticalmente la formulación de las preguntas. Por regla general, con una sesión de dos horas suele ser suficiente para realizar el círculo literario.

Pese a resultar una idea un poco contradictoria con la propia naturaleza del círculo literario, en nuestro caso realizamos un cuestionario de lectura previo a la actividad de unos 10-15 minutos. El objetivo no es tanto evaluar como obtener una retroalimentación rápida. Entre los datos que se consiguen de este test están: si el alumno se ha leído el libro, el grado de comprensión lectora en determinadas partes de la historia y la profundización en la interpretación de la obra. Por otra parte, permite dirigir al alumno hacia los puntos más importantes del relato (en cuanto a estructura, significado, etc.), sirviendo de guía para recordar información relevante que luego se manejará durante la sesión o, incluso, para complementar la explicación posterior de la obra en las clases de literatura.

Un elemento clave dentro del aula es la emoción. De hecho, los últimos estudios en neurociencia revelan que juega un papel fundamental en la fijación de recuerdos y, por lo tanto, es imprescindible tenerlo en cuenta en los procesos de aprendizaje (Ibarrola 2014). Además, como Krashen ya afirmaba en 1982, para adquirir una segunda lengua no solo es necesario entender el input, sino que hay que estar predispuesto a esa entrada. Pero no solo eso, sino que también el ambiente contribuye a la mejora del aprendizaje, creando nuevas neuronas y conexiones nerviosas:

\footnotetext{
${ }^{3}$ La adaptación/traducción es nuestra.
} 
Las neuronas se regeneran como consecuencia de estimulaciones ambientales para adaptarse mejor al medioambiente. Hay que incorporar la "novedad" o repetición justa para crear los automatismos mínimos necesarios, sincronizados temporalmente, y lograr la generación de nuevas conexiones cerebrales entre distintas áreas corticales, así como para estabilizar procesos neurofuncionales básicos. (Ortiz, 2018: 15)

Evidentemente, una actividad como los círculos literarios, fundamentada en el aprendizaje basado en interacciones, requiere una gran carga emocional y voluntad por parte de sus integrantes, al recurrir continuamente a sus experiencias y valoraciones para conectarlas con el libro. Pero asimismo la sorpresa de entrar en el aula y encontrar comida y bebida también refuerza ese componente emocional, dado que funcionan como conectores del relax y la diversión. Se trata de modificar el entorno para pasar de una clase a una reunión (Acaso, 2013: 99-129) y lograr la consideración de esa sesión como algo especial, alejado de la rutina académica diaria.

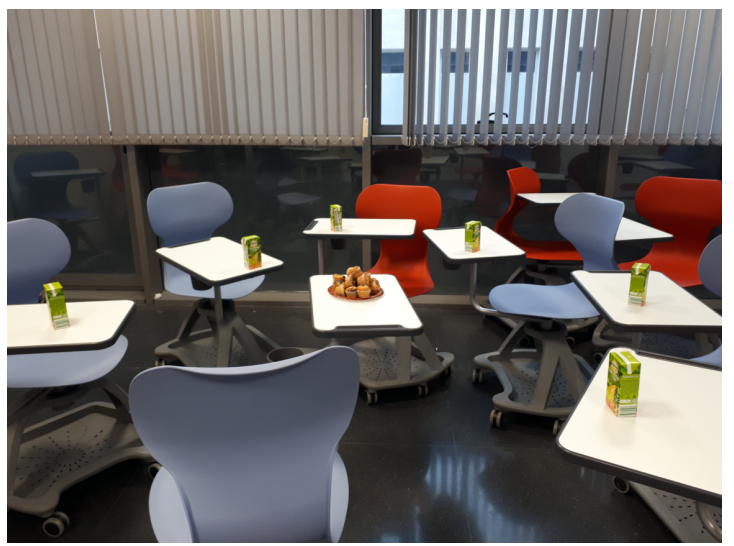

Imagen 1. La comida y la bebida en el aula ayudan a concebir la actividad como algo especial.

Un aspecto esencial durante la actividad es evitar en la medida de lo posible la participación del docente, ya que esto rompería el diálogo entre iguales, característica principal del aprendizaje dialógico y del aprendizaje basado en interacciones. De hecho, debe ser una actividad que ellos controlen para lograr la inhibición que comporta la conversación entre compañeros, de manera que se refuercen los argumentos y no la jerarquía del que habla. Al mismo tiempo, esa «lectura dialógica favorece el desarrollo del pensamiento propio y del pensamiento divergente del alumnado» (Álvarez-Álvarez y Pascual-Díez 2014: 628); en definitiva, de un pensamiento crítico, ya que supone la expresión de sus experiencias, ideas y conocimientos previos a partir de su opinión personal y de las reflexiones estimuladas por las aportaciones del resto del grupo.

\subsection{Posteriormente a la discusión}

La última parte del círculo literario es la concreción de ideas, que puede llevar a cabo el docente (después de haber recogido notas durante la sesión) o los mismos alumnos. Para cualquier actividad en grupo, es aconsejable la figura del "secretario", aquella persona encargada de recoger todas las ideas y temas importantes que se han tratado en su grupo, si bien es cierto que se puede prescindir de esta figura y delegar este papel en todo el equipo. La ventaja de este cargo radica en que permite exponer todos los puntos clave de una manera más sintetizada y organizada, al tiempo que desinhibe al alumno por la asunción de un papel meramente testimonial. También es apto delegarlo en aquellos 
alumnos cuyo rol requiere menos actividad, como por ejemplo el resumidor (summarizer).

Para terminar, se puede plantear una reflexión al alumno, una autoevaluación (cómo valoran su trabajo dentro del grupo) o coevaluación (cómo calificarían la actuación de los diferentes miembros) en forma de ensayo, que sería idóneo para trabajar el texto argumentativo, o rúbricas (previamente consensuadas con ellos). Eso sí, es fundamental trabajar con anterioridad esta última parte en clase para que puedan realizar el trabajo adecuadamente. Buttiler y Massano (2015: 5) proponen algunas tareas posteriores relacionadas con las habilidades propuestas en la taxonomía de Bloom (Bloom, Engelhart, Furst, Hill y Krathwohl, 1956):

1. Listar a los personajes e identificar el escenario (conocimiento).

2. Explicar la relación entre esos personajes y relatar el conflicto principal (comprensión).

3. Hacer uso de la información obtenida anteriormente para completar un mapa conceptual o cuadro (aplicación).

4. Realizar hipótesis o inferencias a partir de alguna información presente en el texto en base a algo que el alumno ya sepa, examinar causas y efectos de las acciones de los personajes (análisis).

5. Diseñar una historieta, redactar una noticia o inventar un juego que consten de información presente en el texto principal (síntesis).

6. Redactar un artículo de opinión acerca de la obra, formulando juicios de valor y justificando (evaluación).

Otra opción es la creación de un blog sobre los círculos literarios, donde se reserve un apartado para comentar los libros de forma individual o en grupo (y, de esta manera, también se amplía el estudio de las tipologías textuales con la utilización de la reseña); o de un mural multimedia, aprovechando los recursos que nos brinda la red (como Glogster, Mural.ly o Padlet, solo por citar algunas opciones), en el que cada círculo haga una puesta en común con toda la información que ha recopilado para preparar los roles (vídeos, fotos, audios, artículos, páginas web, etc.) y la que se ha generado durante la reunión (reflexiones, temas, etc.). De esta manera, la experiencia del libro también se amplifica hacia otras áreas, ya que, como dice Giralt (2009: 117), «la riqueza del texto literario nos permite la multidisciplinariedad (literatura, cine e historia) en el aula de idiomas, elemento que la enriquece notablemente, especialmente en los niveles más avanzados».

En general, existen muchas posibilidades de trabajar actividades complementarias al círculo de lectura que, en cualquier caso, deben adaptarse a la realidad de cada aula y a los objetivos que se hayan marcado.

\section{Evaluación}

Una de las partes más complicadas de la labor docente es sin duda la evaluación, ya que el profesor se enfrenta a la dificultad de encontrar la forma más idónea de calificar una actividad y de la manera más objetiva posible. Por si fuera poco, esto se complica con la aparición de las nuevas metodologías de aprendizaje, como aprendizaje basado en proyectos o aprendizaje colaborativo, donde no solo se tiene que evaluar la actuación de un alumno individualmente, sino como miembro de un grupo con el que participa e interactúa. Daniels (2002: 185-186), en su afán por conservar la idiosincrasia de los clubs de lectura en los círculos literarios, afirma que para evitar que la obsesión por calificar numéricamente la actividad vicie el espíritu de la misma, es preciso observar con 
minuciosidad, dado que esta nos brinda multitud de evidencias para poder evaluar el grado de lectura, aprendizaje y pensamiento del estudiante. De hecho, como afirma Lakey (2010: 111), el trabajo del docente es primordial a la hora de recabar la información necesaria para planificar las actividades complementarias:

Contrary to conventional thinking, the best way to use our knowledge is usually not to present it. Its best use is to inform ourselves how the participants are doing as they pursue their learning goals. Based on this diagnosis, we can devise the additional activities that will enable their success.

Para evaluar los círculos literarios existen multitud de herramientas. Tal y como señala Daniels (2002: 186), es fundamental plantearse una serie de preguntas antes de llevar a cabo la actividad: qué vamos a evaluar, qué pretendemos conseguir cuando observamos, registramos, medimos o juzgamos, e incluso qué teorías y principios guían nuestros esfuerzos. Puede que estas cuestiones resulten obvias, pero muchas veces nos enfrascamos en una determinada forma de evaluar sin tener en cuenta que una misma actividad puede evaluarse de diferente manera en función de la perspectiva que adoptemos. En cualquier caso, es importante conocer las diferentes herramientas con las que contamos para elegir la más adecuada a nuestros objetivos.

Una de las mejores opciones son las rúbricas, que además nos permiten tanto la autoevaluación como la coevaluación (si queremos hacer a los alumnos más partícipes del proceso evaluativo). Vilà y Castellà (2016: 13) señalan que, para que estas funcionen, es preciso que cumplan una serie de requisitos:

1. Brevedad, para poder controlar todas las variables simultáneamente.

2. Flexibilidad, para adecuarlas a cada alumno.

3. Construcción colaborativa con el alumno, no solo con el fin de que este se comprometa con la actividad, sino de que alcance una adecuada reflexión metadiscursiva y pueda controlar su interacción durante la actividad.

4. Diversificación y dosificación, para evaluar en diferentes momentos de la actividad.

5. Concreción y comprensibilidad, con criterios específicos que puedan entender los alumnos.

6. Asunción de objetivos comunicativos y no solo lingüísticos.

7. Desarrollo de observaciones y opiniones, para que el alumno argumente cada una de sus elecciones.

Pero también contamos con otras herramientas que nos permiten constatar el trabajo de los estudiantes. Es el caso de proyectos murales (como hemos comentado anteriormente) o en forma de libro (y en este caso también se podrían aprovechar las opciones que presentan las nuevas tecnologías), donde se recojan todas las aportaciones del grupo con vídeos, referencias web, fotografías, etc.; reuniones individuales con los alumnos donde expliquen oralmente su rol, las dificultades que se han encontrado o aquello que más les ha gustado (aunque estas reuniones también podrían extenderse al grupo completo); o portfolios, para que los alumnos reflexionen sobre lo que han aprendido (Daniels, 2002: 194-199).

Una vez hayamos elegido cómo vamos a evaluar, lo siguiente es determinar el porcentaje que vamos a dar a cada parte. Las opciones son muy diversas, como hemos comentado anteriormente, pero si hemos optado por realizar un control de lectura antes de la actividad, evidentemente para ser coherentes con la naturaleza de los círculos literarios, debemos darle más valor al círculo literario que al examen, por ejemplo: un $30 \%$ frente 
al $70 \%$, sobre todo teniendo en cuenta que la finalidad del control estaba supeditada al propio círculo. Además, con estos valores se reduce el nivel de estrés y ansiedad del estudiante al enfrentarse a un "examen", y se promueve su participación en la reunión (con mayor asignación), que supone una experimentación relativamente libre de la lectura. Por otra parte, estos porcentajes pueden variar si se añaden actividades posteriores a la reunión (como ensayos, reflexiones, etc.). En cualquier caso, lo aconsejable es que el peso de la evaluación recaiga en el círculo literario.

\section{Conclusión}

Para concluir, esta experiencia representa una revalorización de la literatura dentro del aula, dado que utiliza las lecturas no solo como un marcador del grado de comprensión lectora que haya adquirido el estudiante, sino que se convierte en una fuente de cultura, crecimiento personal e interpersonal, estableciendo vínculos y relaciones vivenciales con el libro y los compañeros. Al mismo tiempo, permite al alumno asumir el control de su aprendizaje, de modo que lo supervisa y autorregula a medida que van surgiendo determinadas necesidades (opinión, mediación, etc.), y opera a partir de lo que Vygotsky (1978: 86) denomina zona de desarrollo próxima, puesto que es capaz de resolver problemas con la colaboración de sus iguales. Por otro lado, la asunción de responsabilidades también repercute en la motivación del alumno, junto con otros factores, como son la creación de una atmósfera relajada en el aula y la organización de la actividad a partir de unos objetivos definidos y unos criterios de evaluación consensuados (Lorenzo, 2004: 319-322). Asimismo, al tratarse de un diálogo entre iguales sin la participación expresa del docente y de la expresión de una lectura personal del libro, donde no hay respuestas "correctas/incorrectas", se favorece la desinhibición del estudiante. En definitiva, el círculo literario supone un enriquecimiento para todos sus integrantes, sean alumnos o docentes, que se nutren de las experiencias personales, porque, como dice Rodríguez-Valls (2008: 58), «el enseñar no nace en la cabeza del educador; educar empieza en las experiencias que los estudiantes llevan consigo al aula».

\section{Bibliografía}

ACAso, M. (2013). Reduvolution. Barcelona: Paidós Contextos.

Álvarez, B. (2011). La verdadera historia de los cuentos populares. Madrid: Editorial Morata.

Álvarez-Álvarez, C. y PASCuAl-DíEz, J. (2014). «Aportaciones de un club de lectura escolar a la lectura por placer», El profesional de la información, 23 (6), pp. 625-631.

Bloom, B.S., Engelhart, M.D., Furst, E.J., Hill, W.H. y Krathwohl, D.R. (1956). Taxonomy of educational objective: the classification of educational goals. New York: Longmans.

Boardman Moen, C. (2004). Literature Circles Roles Sheets. Ohio: Lorenz Educational Press.

ButTiler, M.B. y MASSANO, M.C. (2015). «Los círculos literarios en los cursos de adultos de ILE», Puertas abiertas: Revista de la Escuela de Lenguas, 11. Recuperado el 24/6/2020, de http://www.puertasabiertas.fahce.unlp.edu.ar/numeros/numero- 
11/PAn11a08\%20Massano.pdf.

DANIELS, H. (2002). Literature circles. Voice and choice in book clubs and Reading groups. Portsmouth: Stenhouse Publishers.

FURR, M. (2004). «Literature circles for the EFL classroom», Proceedings of the 2003 TESOL Arabia Conference. Dubai: TESOL Arabia.

FURR, M. (2011). Bookworm Club: Reading circle's teacher's handbook. Oxford: Oxford University Press.

FurR, M. (Ed.) (s.f.). Bookworms Club: Stories for Reading Circles. Oxford: Oxford University Press.

GARCÍA, M. V. (2017). El desarrollo de la educación literaria en aprendientes iniciales de ELE. Una propuesta didáctica llevada a las aulas (tesis doctoral). Barcelona: Universitat Autònoma de Barcelona.

GIRALT, M. (2009). «ELE de Literatura: algunas propuestas para llevar la literatura al aula de español». En Actas del I Simposio internacional de literatura española $e$ hispanoamericana del Instituto Cervantes de Brasilia (pp. 116-128). Brasilia: Instituto Cervantes de Brasilia. Recuperado el 25/6/2020, de https://cvc.cervantes.es/ensenanza/biblioteca_ele/publicaciones_centros/PDF/brasilia_2 009/14_giralt.pdf.

HERNÁNDEZ, M. ${ }^{\mathrm{a}}$ del P. (2018). «Acercándonos a Enrique Vila-Matas. Una propuesta didáctica de literatura en ELE». En Actas del XV Encuentro Práctico de ELE del Instituto Cervantes de Nápoles (pp. 11-27). Nápoles: Instituto Cervantes de Nápoles. Recuperado el 25/6/2020,

de https://cvc.cervantes.es/ensenanza/biblioteca_ele/publicaciones_centros/PDF/napoles_2 018/01_hernandez.pdf.

HERnÁNDEZ, M. ${ }^{\mathrm{a}}$ del P. (2019). «Literatura para aprender ELE: Almudena Grandes y su Malena». En Actas del XVI Encuentro Práctico de ELE del Instituto Cervantes de Nápoles (pp. 40-53). Nápoles: Instituto Cervantes de Nápoles. Recuperado el 25/6/2020, de

https://cvc.cervantes.es/ensenanza/biblioteca_ele/publicaciones_centros/PDF/napoles_2 019/04_hernandez.pdf.

IBARROLA, B. (2014). Aprendizaje emocionante. Madrid: Ediciones SM.

INSTITUTO CERVANTES (2002). «Capítulo 5. Las competencias del usuario o alumno». Marco común europeo de referencia para las lenguas: aprendizaje, enseñanza, evaluación. Madrid: Ministerio de Educación, Cultura y Deporte. Recuperado el 09/03/20, de http://cvc.cervantes.es/obref/marco.

KRASHEN, S. (1982). Second Language Acquisition and Second Language Learning. New Jersey: Prentice-Hall.

LAKEY, G. (2010). Facilitating group learning. San Francisco: Jossey-Bass.

LORENZO, F.J. (2004). «La motivación y el aprendizaje de una L2/LE». En Sánchez Lobato, J. y Santos Gargallo, I. (ed.), Vademécum para la formación de profesores (pp. 305-328). Madrid: SGEL.

MARTíneZ, E. (2017), Bajo la piel del lobo, acompañar las emociones con los cuentos 
tradicionales. Barcelona: Editorial Graó.

MiÑANO, J. (2000). «Estrategias de lectura: propuestas prácticas para el aula de E/LE», Carabela, 48, pp. 25-43. Recuperado el 2/7/2020, de https://dialnet.unirioja.es/servlet/articulo?codigo $=7197318$.

Muñoz, C., Valenzuela, J., Avendaño, C., y NúÑEz, C. (2016). «Mejora en la motivación por la Lectura Académica: la mirada de estudiantes motivados», Ocnos, 15 (1), pp. 52-68.

OrTIZ, T. (2018). Neurociencia en la escuela. Madrid: Ediciones SM.

OXFORD UNIVERSITY PRESS. (s.f). [Oxford Bookworms Club Reading Circles]. Recuperado el 1/7/2020, de https://elt.oup.com/teachers/readingcircles/

RODRÍGUEZ-VALLS, F. (2008). «Círculos literarios, cooperativas de lectura: leer para transformar», Lectura y vida: Revista Latinoamericana de Lectura, 29 (2), pp. 56-63. Recuperado el 1/6/2020, de https://dialnet.unirioja.es/servlet/articulo?codigo=2799701.

SÁNCHEZ, M. (2004). «El papel de la literatura en la enseñanza de español». En Actas del I Encuentro Práctico de ELE del Instituto Cervantes de Manila (pp. 72-83). Manila: Instituto Cervantes de Manila. Recuperado el 2/7/2020, de https://cvc.cervantes.es/ensenanza/biblioteca_ele/publicaciones_centros/PDF/manila_20 04/09_sanchez.pdf.

SoLÉ, I. (1992). Estrategias de lectura. Barcelona: Editorial Grao.

VAlls, R., Soler, M. y Flecha, R. (2008). «Lectura dialógica: interacciones que mejoran y aceleran la lectura», Revista Iberoamericana de Educación, 46 (1), pp. 71-87. Recuperado el 9/3/2020, de https://dialnet.unirioja.es/servlet/articulo?codigo=2661637.

Vygotsky, L. S. (1978). Mind in Society: The Development of Higher Psychological Processes. Cambridge, Massachusetts: Harvard University Press.

VILA, M. y CASTEllà, J.M. (2016). «La evaluación de la lengua oral. Retos y alternativas», Textos. Didáctica de la Lengua y la Literatura, 73, pp. 7-18. 